\title{
Reduced DNA methylation of the oxytocin receptor gene is associated with obsessive- compulsive disorder
}

\author{
Chun II Park ${ }^{1,3}$, Hae Won Kim ${ }^{3,4}$, Sumoa Jeon ${ }^{3}$, Jee In Kang ${ }^{2,3^{*}}$ and Se Joo Kim ${ }^{2,3^{*}}$ (D)
}

\begin{abstract}
Background: Oxytocin is an important neuromodulator involved in cognition and socio-emotional processing that exerts its central activities via oxytocin receptors. Epigenetic alterations in the oxytocin receptor gene (OXTR) may be a molecular mechanism in the pathogenesis of obsessive-compulsive disorder (OCD). This study investigated the association between OXTR DNA methylation and the OCD status of a Korean population.

Results: Quantitative leukocyte DNA methylation levels of three cytosine-phosphate-guanine (CpG) sites in the 5' untranslated region (UTR) of OXTR exon 2 and eight CpG sites within OXTR exon 3 were analyzed using the pyrosequencing method in 151 patients with OCD (including 45 drug-naïve patients) and 108 healthy controls. DNA methylation levels were compared between the groups using multiple analyses of covariance separately by sex after controlling for age and educational level. Patients with OCD showed significantly lower methylation levels at CpG1 and CpG2 sites on the UTR of OXTR exon 2 than those of healthy controls for both sexes. In a subset of 45 drug-naiive patients with OCD, the DNA methylation levels also remained significantly lower than those in the controls and their CpG1 methylation levels were significantly negatively associated with the ordering symptom dimension.
\end{abstract}

Conclusions: Our findings suggest that epigenetic OXTR alterations may affect the pathophysiology of OCD. The potential role of the oxytocin system in OCD development and treatment warrants further investigation.

Keywords: Obsessive-compulsive disorder, Oxytocin, OXTR, Epigenetics, DNA methylation

\section{Background}

Oxytocin is an important neuropeptide that regulates social behaviors and is involved in various cognitive and emotional processes $[1,2]$. Since oxytocin exerts its central activities via oxytocin receptors $(O X T R)$ in the brain, studying alterations in OXTR is of great importance for understanding the molecular mechanisms underlying the pathogenesis of psychiatric conditions related to socioemotional processing [3-5]. DNA methylation, an epigenetic mechanism by which cells dynamically control gene expression without changing the DNA sequence, has

\footnotetext{
* Correspondence: jeeinkang@yuhs.ac; kimsejoo@yuhs.ac

${ }^{2}$ Department of Psychiatry, Yonsei University College of Medicine, Yonsei-ro 50-1, Seodaemun-gu, Seoul 03722, Republic of Korea

Full list of author information is available at the end of the article
}

emerged as a crucial mechanism underlying the interaction between genetic background and environmental factors in the development of psychiatric disorders [6]. It has been reported that differential OXTR DNA methylation is involved in various psychological functions relevant to psychopathology including callous-unemotional traits [7], social perception [8], and resilience [9] and is also involved in psychiatric illnesses such as autism spectrum disorder (ASD) [4], postpartum depression [10], social anxiety disorder [11], and early-stage schizophrenia [12].

Epigenetic alterations in the OXTR gene are a promising candidate marker for mediating genetic susceptibility to obsessive-compulsive disorder (OCD), which is characterized by recurrent and disturbing obsessions and repetitive compulsive behaviors. OCD is known to develop 
via complex interactions between genetic and environmental factors [13]. The oxytocin system has been implicated as having a role in the pathogenesis and treatment of OCD [14]; however, little research has been carried out on the epigenetics of OXTR in OCD. Studies of the oxytocin system in OCD have primarily focused on changes in oxytocin levels $[15,16]$ and the DNA sequence without considering the role of epigenetic factors [17]. Recently, an epigenetic study with a small sample size showed that OXTR hypermethylation was associated with OCD susceptibility and symptom severity [18]. Given the emerging role of OXTR in OCD pathogenesis, investigating epigenetic $O X T R$ alterations may be important for understanding the molecular mechanisms underlying $\mathrm{OCD}$ pathogenesis and discovering better biomarkers for OCD development and progression.

In this study, we investigated differences in the leukocyte DNA methylation level of OXTR between patients with OCD and healthy controls. To exclude the potential effects of medication on DNA methylation in patients with OCD, we examined whether the results of the whole cohort were confirmed in a subgroup of drugnaïve patients with OCD. Moreover, we examined how DNA methylation levels at specific OXTR cytosinephosphate-guanine ( $\mathrm{CpG}$ ) sites were associated with symptom severity and OCD dimensions.

\section{Results}

\section{Demographics and clinical characteristics}

The demographics and clinical features of each group are presented in Table 1 . There were significant differences in the DNA methylation levels of several OXTR1 and OXTR2 CpG sites between men and women. Women in the control group showed higher DNA methylation levels at OXTR1 CpG1 $(t=-2.40, p=$ $0.018)$ and CpG2 $(t=-2.83, p=0.006)$. Similarly, women in the OCD group showed hypermethylation at all three OXTR1 sites compared to the men: CpG1 $(t=$ -2.08, $p=0.039)$, CpG2 $(t=-2.80, p=0.006)$, and CpG3 $(t=-2.27, p=0.025)$. For OXTR2, there was no significant difference between women and men in either the control or patient groups.

\section{Group differences in OCD status due to OXTR DNA methylation}

As shown in Table 2, multivariate analysis of covariance (MANCOVA) revealed that OCD status had a significant overall effect on DNA methylation levels in both men (Wilks $\lambda=0.822, F_{(4,161)}=8.693, p<0.001$ ) and women (Wilks $\lambda=0.736, \mathrm{~F}_{(4,84)}=7.525, p<0.001$ ). Post hoc comparisons using Bonferroni-adjusted alpha for the four dependent variables indicated that patients with OCD had significantly lower methylation levels at OXTR1 CpG1 and CpG2 sites and no significant difference at OXTR1 CpG3 compared to healthy controls for both men and women. No significant findings were found for OXTR2 methylation levels.

When the same analysis was carried out on a subset of drug-naïve subjects to exclude the potential effects of medication on DNA methylation levels, the significant OXTR findings were still significant in both sexes. MANCOVA revealed significant overall effects on the affective status of OCD in men (Wilks $\lambda=0.840, F_{(4,81)}=3.843$, $p=0.007$ ) and women (Wilks $\lambda=0.666, F_{(4,58)}=7.285$, $p<0.001$ ) in OXTR1, but not in OXTR2. Drug-naïve patients with OCD showed significantly lower levels of DNA methylation at CpG1 (men: $F=7.642, p=0.007$, $\eta_{\mathrm{p}}^{2}=0.083$; women: $\left.F=10.389, p<0.001, \eta_{\mathrm{p}}^{2}=0.193\right)$ and CpG2 (men: $F=14.633, p<0.001, \eta_{\mathrm{p}}^{2}=0.148$; women: $\left.F=5.908, p=0.002, \eta_{\mathrm{p}}{ }^{2}=0.147\right)$ sites than the healthy controls in both sexes (Table 3). There was no significant difference in the DNA methylation status between drug-naïve and drug-treated patients with $\mathrm{OCD}$ for all the CpG sites in each sex (Supplementary Table S1).

\section{Relationships between OXTR methylation and clinical characteristics of $O C D$}

Partial correlation analyses among the drug-naïve patients with OCD revealed that OCD symptom severity

Table 1 Demographics and clinical features of participants

\begin{tabular}{|c|c|c|c|c|c|c|}
\hline & \multicolumn{2}{|l|}{ Men } & \multirow[b]{2}{*}{$p^{b}$} & \multicolumn{2}{|l|}{ Women } & \multirow[b]{2}{*}{$p^{b}$} \\
\hline & $\overline{\mathrm{OCD}^{\mathrm{a}}(n=112)}$ & $\mathrm{HC}^{\mathrm{a}}(n=56)$ & & $\mathrm{OCD}^{\mathrm{a}}(n=39)$ & $\mathrm{HC}^{\mathrm{a}}(n=52)$ & \\
\hline Age & $21.9 \pm 2.2$ & $21.5 \pm 3.5$ & 0.385 & $21.8 \pm 2.2$ & $21.1 \pm 3.6$ & 0.269 \\
\hline Education, year & $12.7 \pm 1.7$ & $13.5 \pm 1.7$ & 0.006 & $13.1 \pm 1.8$ & $13.6 \pm 1.9$ & 0.26 \\
\hline Age of onset & $14.3 \pm 4.1$ & & & $14.7 \pm 4.0$ & & \\
\hline Duration of illness, year & $7.6 \pm 4.5$ & & & $7.0 \pm 3.9$ & & \\
\hline MADRS & $20.6 \pm 9.8$ & & & $19.7 \pm 9.7$ & & \\
\hline Y-BOCS & $25.0 \pm 6.9$ & & & $25.9 \pm 6.5$ & & \\
\hline
\end{tabular}

OCD obsessive-compulsive disorder, HC healthy control, MADRS Montgomery-Asberg Depression Rating Scale, Y-BOCS Yale-Brown Obsessive-Compulsive Scale ${ }^{a}$ Mean \pm standard deviation

${ }^{\mathrm{b}}$ Independent samples $t$ test 
Table 2 Results of MANCOVA of OXTR DNA methylation between patients with OCD and healthy controls for men and women

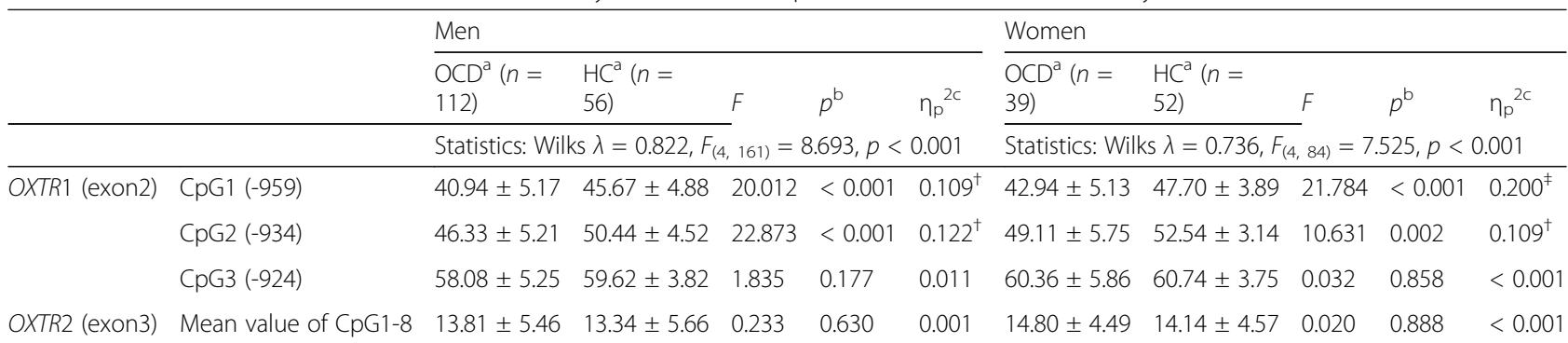

MANCOVA demonstrated significant between-group differences after controlling for age and education

MANCOVA multivariate analysis of covariance, OCD obsessive-compulsive disorder, $H C$ healthy control

${ }^{a}$ Mean \pm standard deviation of raw data

${ }^{\mathrm{b}}$ Statistical significance was set at $p<0.0125$ after Bonferroni correction for the $4 \mathrm{CpG}$ sites

${ }^{C}$ Effect size was calculated using partial eta squared and interpreted according to the rule of Miles and Shevlin (2001)

${ }^{\dagger}$ Medium effect $(>0.06)$

${ }^{\ddagger}$ Large effect $(>0.14)$

measured by Yale-Brown Obsessive Compulsive Scale (Y-BOCS) had no significant association with DNA methylation levels at OXTR1 CpG1 and CpG2, which showed a significant between-group difference $(r=0.146$ and $p=0.358$ for CpG1; $r=-0.211$ and $p=0.18$ for CpG2). Among the six OC symptom dimensions (washing, obsessing, hoarding, ordering, checking, and neutralizing) measured by the Korean version of ObsessiveCompulsive Inventory-Revised (OCI-R-K), ordering dimension scores were significantly and negatively correlated with DNA methylation at the CpG1 site $(r=-$ $0.422, p=0.005$; Fig. 1$)$. Conversely, CpG1 DNA methylation levels were not significantly associated with any OCD dimensions.

\section{Discussion}

The role of epigenetic OXTR regulation via DNA methylation in psychiatric disorders such as OCD is a promising and relatively new field of investigation. The key finding of this study was that both male and female patients with OCD showed significantly altered OXTR1 methylation status. These significant findings were also confirmed in a subset of drug-naïve patients with OCD, suggesting that OXTR1 epigenetic alterations may play a crucial role in the pathogenesis of OCD and act as a molecular mechanism underlying the development and recovery of OCD, possibly via interactions with genetic predisposition and environmental factors.

In this study, patients with OCD exhibited DNA hypomethylation (which may indicate higher OXTR expression) at CpG1 (Chr3: 8,810,833) and CpG2 (Chr3: 8,810, 808 ) in the $5^{\prime}$ untranslated region (UTR) of OXTR exon 2 compared to healthy controls. To date, only one study with a small sample size (42 patients with OCD vs. 31 controls) has reported OXTR methylation in OCD, finding that hypermethylation of specific $\mathrm{CpG}$ sites in OXTR exon 3 was associated with OCD disease status, correlating positively with $\mathrm{OC}$ symptom severity and negatively with depressive scores [18]. Although various differences between our study and theirs, including age and target CpG sites, make direct comparison difficult, their findings indicated that the associations of OXTR methylation

Table 3 Results of MANCOVA of DNA methylation at OXTR CpG sites between drug-naïve patients with OCD and healthy controls for men and women

\begin{tabular}{|c|c|c|c|c|c|c|c|c|c|c|c|}
\hline & & \multicolumn{5}{|l|}{ Men } & \multicolumn{5}{|l|}{ Women } \\
\hline & & $\begin{array}{l}\mathrm{OCD}^{\mathrm{a}}(n= \\
32)\end{array}$ & $\begin{array}{l}\mathrm{HC}^{\mathrm{a}}(n= \\
56)\end{array}$ & $F$ & $p^{\mathrm{b}}$ & $\eta_{p}^{2 c}$ & $\begin{array}{l}\mathrm{OCD}^{\mathrm{a}}(n= \\
13)\end{array}$ & $\begin{array}{l}\mathrm{HC}^{\mathrm{a}}(n= \\
52)\end{array}$ & $F$ & $p^{b}$ & $\eta_{p}^{2 c}$ \\
\hline & & \multicolumn{5}{|c|}{ Statistics: Wilks $\lambda=0.840, F_{(4,81)}=3.843, p=0.007$} & \multicolumn{5}{|c|}{ Statistics: Wilks $\lambda=0.666, F_{(4,58)}=7.285, p<0.001$} \\
\hline \multirow[t]{3}{*}{ OXTR1 (exon2) } & CpG1 (-959) & $41.49 \pm 5.66$ & $45.67 \pm 4.88$ & 7.642 & 0.007 & $0.083^{\dagger}$ & $42.75 \pm 5.96$ & $47.70 \pm 3.89$ & 10.389 & $<0.001$ & $0.193^{\ddagger}$ \\
\hline & CpG2 (-934) & $46.41 \pm 4.73$ & $50.44 \pm 4.52$ & 14.633 & $<0.001$ & $0.148^{\ddagger}$ & $48.87 \pm 3.72$ & $52.54 \pm 3.14$ & 5.908 & 0.002 & $0.147^{\ddagger}$ \\
\hline & CpG3 $(-924)$ & $57.41 \pm 4.45$ & $59.62 \pm 3.82$ & 6.044 & 0.016 & 0.067 & $61.49 \pm 6.12$ & $60.74 \pm 3.75$ & 0.695 & 0.368 & 0.013 \\
\hline OXTR2 (exon3) & Mean value of CpG1-8 & $14.32 \pm 6.31$ & $13.34 \pm 5.66$ & 0.388 & 0.535 & 0.005 & $13.79 \pm 4.27$ & $14.14 \pm 4.57$ & 0.274 & 0.599 & 0.005 \\
\hline
\end{tabular}

MANCOVA demonstrated significant between-group difference after controlling for age and education

MANCOVA multivariate analysis of covariance, $O C D$ obsessive-compulsive disorder, $H C$ healthy control

${ }^{a}$ Mean \pm standard deviation of raw data

${ }^{\text {b }}$ Statistical significance was set at $p<0.0125$ after Bonferroni correction for 4 CpG sites

'Effect size was calculated using partial eta squared and interpreted according to the rule of Miles and Shevlin (2001)

${ }^{\dagger}$ Medium effect $(>0.06)$

${ }^{\ddagger}$ Large effect $(>0.14)$ 


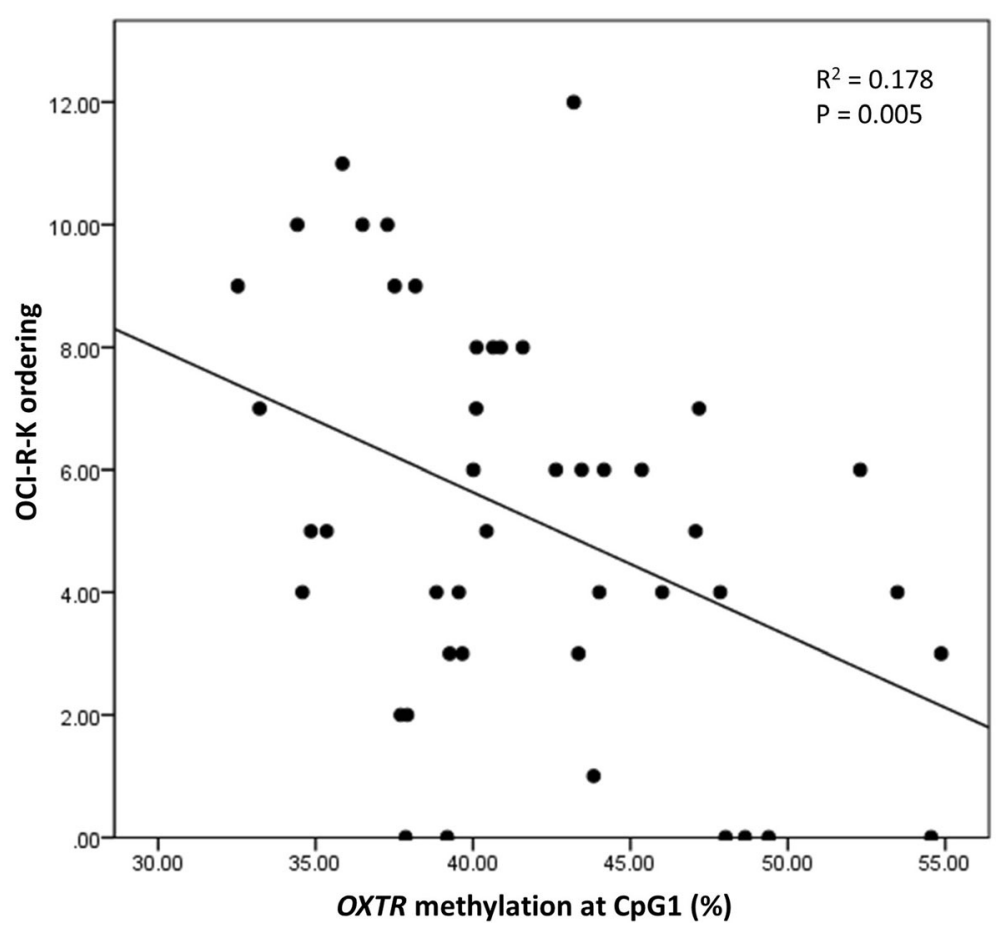

Fig. 1 Correlation between DNA methylation levels at OXTR1 CpG1 and ordering symptom severity in drug-naiive patients with OCD $(n=45)$. OXTR, oxytocin receptor gene; OCl-R-K, obsessive-compulsive inventory-revised-Korean; OCD, obsessive-compulsive disorder

acted in the opposite direction to our findings. Compared to the previous study, we believe that our study has several advantages, including a larger sample size and a more reliable OCD recruitment method (tertiary hospital vs. advertisement), and our findings showed consistent results in both male and female subjects as well as in drug-naïve patients. Furthermore, several studies have reported similar findings from fields of psychiatry other than OCD; for instance, the same direction of OXTR hypomethylation was observed in a clinical study of ASD [19]. A recent study of early-stage schizophrenia also demonstrated that hypomethylation status at the same genomic OXTR position as our significant results was associated with susceptibility to schizophrenia and anhedonia-asociality in women [12]. A systematic narrative review of OXTR DNA methylation in human socioemotional functioning suggested that while OXTR hypermethylation may play a role in the general impairment of socio-emotional functioning, OXTR hypomethylation may play a role in specific patterns of impairment related to psychiatric disorders, such as anxiety disorders [3]. As proposed by Ziegler et al. who demonstrated the relationship between OXTR exon 3 hypomethylation and social anxiety, OXTR hypomethylation findings suggest that higher OXTR expression might act as compensatory upregulation for reduced oxytocin levels [11]. However, previous studies on OCD could not conclude that altered oxytocin level in cerebrospinal fluid was associated with disease status $[15,20]$. Interestingly, Marroni et al. argued that the oxytocin receptor's failure to respond to oxytocin activation might be involved in the continuation of compulsive behavior in their study which investigated an experimental animal model of hypergrooming behavior [21]. In addition, some basic studies suggested that inhibited neuronal activity induced DNA demethylation of specific receptors [22, 23]. The relationship between methylation status and dysfunctional receptor properties or signaling in oxytocin system is still unknown, and it may be an alternative area to investigate. Therefore, it is crucial to elucidate the mechanisms of interaction between OXTR methylation and oxytocin receptor dysfunction in patients with OCD in future studies. Since there is currently very limited evidence on epigenetic OXTR regulation in psychiatric fields including OCD and the direction of the association has been reported to show mixed findings, further longitudinal studies or preclinical studies are required to better establish the role of OXTR methylation in OCD pathogenesis to determine whether altered OXTR methylation status confers vulnerability to OCD development or is a consequence of OCD.

Given the distinct neural correlates of OC symptom dimensions [24], we examined whether the DNA methylation levels at the OXTR1 CpG sites that showed a significant between group-difference were associated with certain symptom dimensions in drug-naïve patients with 
OCD. Among the six symptom dimensions assessed by the OCI-R-K, DNA methylation levels at OXTR1 CpG1 were found to be inversely associated with ordering dimension scores in drug-naïve patients with OCD [2527]. Patients with high ordering dimension scores, characterized by ordering and arranging their surroundings and ensuring that objects are arranged in exactly the right way, have been found to have different characteristics, such as an earlier onset age, a more familial OCD form, impaired set-switching abilities, and a dissociable neural system [24, 28, 29]. Epigenetic OXTR regulation may be a molecular mechanism underlying the clinical and biological differences in the ordering dimension. Although it is unclear how OXTR methylation relates specific to the symptom dimension, it is possible that the ordering/arranging symptom dimension includes shared clinical features of OCD and ASD [25-27] that might be modulated by OXTR methylation. This will need to be elucidated in further studies.

This study has several limitations to consider. Firstly, since we cannot measure the methylation status of human brain tissue directly in a clinical study, we assumed that the DNA methylation status of the peripheral blood reflected that of the CNS. Although altered OXTR DNA methylation in the blood has been shown to be significantly associated with gene expression in brain tissue [4] and the neural response of social and emotional processing $[8,30,31]$, their relationship across tissues has not yet been clearly established. Secondly, although OXTR methylation status have been observed to be associated with OXTR mRNA expression [4], hormone analysis of circulating oxytocin levels and mRNA expression of oxytocin and OXTR were not evaluated in this study. Thus, we cannot determine the functional significance of the CpGs studied and their relationship with oxytocin levels. Thirdly, since different genotypes may affect DNA methylation, we could not completely rule out the potential effect of different genotypes of OXTR gene on the present results. However, we previously reported that there was no significant difference in the genotype distributions and the haplotype frequencies for 10 common SNPs (rs1042778, rs237885, rs237887, rs2268490, rs4686301, rs2268493, rs2254298, rs13316193, rs53576, and rs2268498) on OXTR gene between patients with OCD $(N=615)$ and healthy controls $(N=581)$ [17]. When we additionally examined in a subset of the present sample with OCD $(n=129)$ whether the different genotypes of the 10 SNPs of OXTR gene have different methylation levels of OXTR1, there was no association between OXTR SNPs and methylation levels in the CpGs of OXTR1 (all $p>0.05$ ). Fourthly, we did not consider potential confounders that may affect the DNA methylation status of the OXTR gene, such as cigarette smoking status [32], heterogeneity of white blood cell types [33], and exposure to environmental factors such as diet, maternal care, and lifestyle [34]. Hence, further research that considers these potential confounders and gene-environment interactions are warranted to confirm our results. Fifthly, we cannot conclude whether the epigenetic OXTR alterations reflect specific OCD factors or shared traits with other psychiatric disorders related to socio-emotional problems and repetitive behaviors. Lastly, our study had a cross-sectional design; thus, conclusions cannot be drawn regarding the causal direction of the relationships between epigenetic alterations and OCD. To better establish the role of OXTR methylation and causal relationships in the pathogenesis and course of OCD, further longitudinal studies are needed.

\section{Conclusions}

The present study showed altered DNA methylation status of OXTR1 in both male and female patients with OCD as well as in a subset of drug-naïve OCD patients, compared to healthy controls. Our findings suggest that epigenetic alterations of OXTR may exert an effect on the pathophysiology of OCD. The potential role of OXTR in the development and treatment of OCD warrants further investigation.

\section{Methods \\ Participants}

A total of 151 patients (112 men, 39 women) with OCD were recruited from a specialized OCD outpatient clinic at Severance Hospital of Yonsei University Health System (Seoul, Republic of Korea), a tertiary care hospital. All patients were referred from primary care for OCD and were assessed by trained psychiatrists using the Structured Clinical Interview for DSM-IV-TR [35] to confirm the existence of current or past psychiatric disorders. The demographic and clinical information of the patients were also systematically assessed. Patients were excluded if they met the following criteria: psychiatric disorders with psychotic symptoms, other anxiety disorders, substance dependence, mental retardation (as defined by DSM-IV), history of major head trauma, or current major somatic or neurological disorders. For the control group, we recruited 108 healthy subjects (56 men, 52 women) using posters and online advertisements. According to the DSM-IV-TR diagnostic criteria, subjects with a current or previous lifetime history of any psychiatric disorders were excluded from the study. All participants provided written informed consent according to procedures approved by the Severance Hospital Institutional Review Board and all methods conformed to the approved guidelines. 
Measurement of OC symptoms and clinical characteristics Clinical symptoms were assessed by a trained psychologist using the 10-item Y-BOCS [36] for OC symptom severity and the Montgomery-Åsberg Depression Rating Scale (MADRS) [37] for depression severity. We also used the OCI-R-K $[38,39]$ to assess the severity of OC symptoms via dimensions including washing, obsessing, hoarding, ordering, checking, and neutralizing.

\section{Pyrosequencing procedures}

Three CpG sites in the 5' UTR of OXTR exon 2 (OXTR1) and eight $C p G$ sites in the protein coding region of $O X T R$ exon 3 (OXTR2) were targeted based on previous studies: OXTR1 (Chr3: 8,810,729-8,810,845; GRCh37/hg19) [30] and OXTR2 (Chr3: 8,809,281-8,809,534; GRCh37/hg19) $[11,40]$. The OXTR1 included the CpG site -934 (relative to translation start site) in which elevated DNA methylation level was reported to be related to decreased expression of OXTR in temporal cortex tissue of autistic males
[4], and the methylation level was revealed to be associated with brain activity in dorsal anterior cingulate cortex which is important in OCD pathophysiology [30]. We also targeted CpG sites in OXTR2 which were reported to have significant relationships with response to social stress in social phobia or non-clinical subjects $[11,40]$, considering that several studies suggested an impairment of social cognition in patients with OCD $[41,42]$. These selected CpG sites are shown in Fig. 2.

Genomic DNA was extracted from whole blood using standard techniques by DNA Link, Inc. (Seoul, Republic of Korea). The pyrosequencing procedure was used for DNA methylation analysis by Genomictree, Inc. (Daejeon, Republic of Korea). The bisulfite conversion was conducted using the EZ DNA Methylation-Lightning kit (Zymo Research, CA, USA). The target regions were amplified using polymerase chain reaction (PCR) with converted DNA, and the PCR was conducted in accordance to the general guidelines of pyrosequencing.

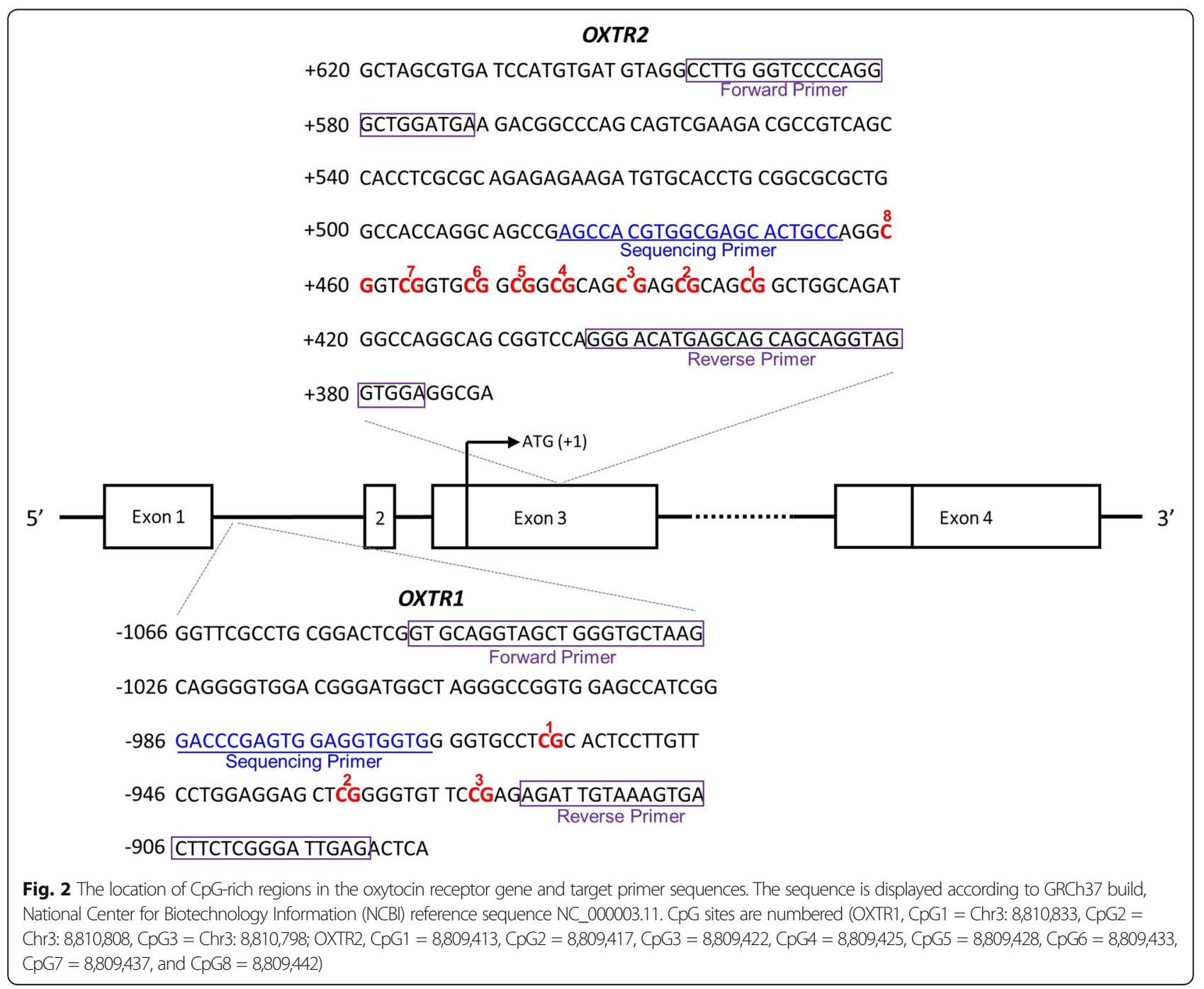


Successful PCR products were confirmed using electrophoresis on a $2 \%$ agarose gel. The pyrosequencing was performed using a PyroMark ID system with the Pyro Gold reagents kit (Qiagen, Hilden, Germany). To control for the internal quality of completeness of bisulfite treatment, the analysis of a non-CpG cytosine was included during pyrosequencing. More detailed information of these procedures have been described elsewhere [12].

\section{Statistical analysis}

All data analysis was conducted using SPSS 25 software (IBM Corp., Armonk, NY, USA). Descriptive statistics were calculated for demographic and clinical variables, with continuous variables presented as the mean \pm standard deviation (SD). Differences in the demographic data between patients with OCD and healthy controls were assessed using $t$ tests. Data were examined for normality by visual inspection of their histograms and the Shapiro-Wilk test. For variables with non-normal distribution, normal score with the Blom method [43] was used for group comparisons. Since the DNA methylation levels were not normally distributed, Blom transformation was applied. The distributions of the raw data of methylation levels of individual CpGs (Supplementary Fig. 1) and a description of the normalization process are included in the supplementary material.

MANCOVA was conducted to evaluate the influence of OCD status on OXTR1 and OXTR2 DNA methylation levels with covariates of age and education level. Given that oxytocin system function is sexually dimorphic [4446], we conducted the analyses separately for men and women. Since DNA methylation levels at the eight OXTR2 CpG sites were highly intercorrelated (Cronbach's alpha, men with OCD 0.99, women with OCD 0.985, control men 0.981, control women 0.975 ), the average methylation level of the eight CpG sites was used as a variable for the analyses. Thus, we used the DNA methylation levels at four CpG sites (three OXTR1 CpGs and one averaged value for 8 OXTR2 CpGs) as dependent variables in the MANCOVA model. Additionally, to rule out potential effects of medication, we compared the DNA methylation status between drug-naïve patients and healthy controls, and drug-naïve and drug-treated patients using MANCOVA. Statistical significance was adjusted for the multiple comparisons of the four $\mathrm{CpG}$ sites using the Bonferroni method $(\alpha=0.0125)$.

To investigate the relationships between OXTR DNA methylation levels and clinical OCD characteristics as measured by Y-BOCS and OCI-R-K, partial correlation coefficients were calculated, controlling for the potential confounding effects of sex, age, and MADRS score. Partial correlation coefficients were used for drug-naïve patients with $\mathrm{OCD}(n=45)$ to exclude the potential effects of medication.

\section{Supplementary information}

Supplementary information accompanies this paper at https://doi.org/10. 1186/s13148-020-00890-w.

\section{Additional file 1: Supplementary material. Evaluation of Normality for DNA methylation levels of CpG sites. Supplementary Fig. 1. Histograms of the variables of the CpG sites for each group in males and females. Table S1. Results of MANCOVA ${ }^{a}$ of DNA methylation at OXTR CpG sites between drug-naive patients with OCD and drug-treated pa- tients with OCD for men and women. Table S2. Partial correlation ${ }^{\mathrm{a}}$ be- tween DNA methylation levels at OXTR1 CPG1 and CpG2 and OC symptom dimensions based on OCI-R-K in drug-naïve patients with OCD $(n=45)$}

\section{Abbreviations}

OXTR: Oxytocin receptor gene; OCD: Obsessive-compulsive disorder; CpG site: Cytosine-phosphate-guanine site; UTR: Untranslated region; ASD: Autism spectrum disorder; MANCOVA: Multivariate analysis of covariance; $Y$ -

BOCS: Yale-Brown Obsessive Compulsive Scale; OC symptom: Obsessivecompulsive symptom; MADRS: Montgomery-Åsberg Depression Rating Scale; OCI-R-K: Korean version of Obsessive-Compulsive Inventory-Revised; OXTR1: $5^{\prime}$ UTR of OXTR exon 2; OXTR2: OXTR exon 3; PCR: Polymerase chain reaction

\section{Acknowledgements}

Not applicable.

\section{Authors' contributions}

SK and JK conceived and planned the study. SK and SJ contributed to the data collection and management. CP, HK, and JK performed the statistical analyses and interpreted the findings. CP, JK, and SK wrote the manuscript. HK provided scientific input and helped edit the manuscript. All authors contributed to and have approved the final manuscript.

\section{Funding}

This work was supported by the National Research Foundation of Korea (NRF) grant funded by the Korea government (NRF-2018R1A2B2007714). The funding source did not give any influences on the study design, data collection, analysis and interpretation of data, the writing of the report, and the decision to submit the paper for publication.

\section{Availability of data and materials \\ Not applicable.}

\section{Ethics approval and consent to participate}

All participants provided written informed consent according to procedures approved by the Severance Hospital Institutional Review Board (4-2015-0655) and all methods conformed to the approved guidelines.

\section{Consent for publication}

Not applicable.

\section{Competing interests}

The authors declare that they have no competing interests.

\section{Author details}

${ }^{1}$ Department of Psychiatry, CHA Bundang Medical Center, CHA University, Seongnam, Republic of Korea. ${ }^{2}$ Department of Psychiatry, Yonsei University College of Medicine, Yonsei-ro 50-1, Seodaemun-gu, Seoul 03722, Republic of Korea. ${ }^{3}$ Institute of Behavioral Science in Medicine, Yonsei University College of Medicine, Seoul, Republic of Korea. ${ }^{4}$ Department of Medical Education, Yonsei University College of Medicine, Seoul, Republic of Korea.

Received: 24 March 2020 Accepted: 23 June 2020

Published online: 06 July 2020

\section{References}

1. Bachner-Melman R, Ebstein RP. The role of oxytocin and vasopressin in emotional and social behaviors. Handb Clin Neurol. 2014;124:53-68.

2. Neumann ID. Brain oxytocin: a key regulator of emotional and social behaviours in both females and males. J Neuroendocrinol. 2008;20(6):858-65. 
3. Maud C, Ryan J, McIntosh JE, Olsson CA. The role of oxytocin receptor gene (OXTR) DNA methylation (DNAm) in human social and emotional functioning: a systematic narrative review. BMC Psychiatry. 2018;18(1):154.

4. Gregory SG, Connelly JJ, Towers AJ, Johnson J, Biscocho D, Markunas CA, et al. Genomic and epigenetic evidence for oxytocin receptor deficiency in autism. BMC Med. 2009;7:62.

5. Feldman R, Monakhov M, Pratt M, Ebstein RP. Oxytocin pathway genes: evolutionary ancient system impacting on human affiliation, sociality, and psychopathology. Biol Psychiatry. 2016;79(3):174-84.

6. Caspi A, Moffitt TE. Gene-environment interactions in psychiatry: joining forces with neuroscience. Nat Rev Neurosci. 2006;7(7):583-90.

7. Cecil CA, Lysenko LJ, Jaffee SR, Pingault J-B, Smith RG, Relton $C L$, et al. Environmental risk, oxytocin receptor gene (OXTR) methylation and youth callous-unemotional traits: a 13-year longitudinal study. Mol Psychiatry. 2014;19(10):1071-7.

8. Puglia MH, Lillard TS, Morris JP, Connelly JJ. Epigenetic modification of the oxytocin receptor gene influences the perception of anger and fear in the human brain. Proc Natl Acad Sci U S A. 2015;112(11):3308-13.

9. Milaniak I, Cecil CAM, Barker ED, Relton CL, Gaunt TR, McArdle W, et al. Variation in DNA methylation of the oxytocin receptor gene predicts children's resilience to prenatal stress. Dev Psychopathol. 2017;29(5):1663-74.

10. Bell AF, Carter C, Steer CD, Golding J, Davis JM, Steffen AD, et al. Interaction between oxytocin receptor DNA methylation and genotype is associated with risk of postpartum depression in women without depression in pregnancy. Front Genet. 2015;6:243.

11. Ziegler C, Dannlowski U, Brauer D, Stevens S, Laeger I, Wittmann H, et al. Oxytocin receptor gene methylation: converging multilevel evidence for a role in social anxiety. Neuropsychopharmacology. 2015;40(6):1528-38.

12. Bang M, Kang JI, Kim SJ, Park JY, Kim KR, Lee SY, et al. Reduced DNA methylation of the oxytocin receptor gene is associated with anhedoniaasociality in women with recent-onset schizophrenia and ultra-high risk for psychosis. Schizophr Bull. 2019.

13. Grisham JR, Anderson TM, Sachdev PS. Genetic and environmental influences on obsessive-compulsive disorder. Eur Arch Psychiatry Clin Neurosci. 2008;258(2):107-16.

14. Cochran DM, Fallon D, Hill M, Frazier JA. The role of oxytocin in psychiatric disorders: a review of biological and therapeutic research findings. Harv Rev Psychiatry. 2013;21(5):219-47.

15. Leckman JF, Goodman WK, North WG, Chappell PB, Price LH, Pauls DL, et al. Elevated cerebrospinal fluid levels of oxytocin in obsessive-compulsive disorder. Comparison with Tourette's syndrome and healthy controls. Arch Gen Psychiatry. 1994;51(10):782-92.

16. Marazziti D, Baroni S, Giannaccini G, Catena-Dell'Osso M, Piccinni A, Massimetti $G$, et al. Plasma oxytocin levels in untreated adult obsessivecompulsive disorder patients. Neuropsychobiology. 2015;72(2):74-80.

17. Kang Jl, Kim HW, Kim CH, Hwang EH, Kim SJ. Oxytocin receptor gene polymorphisms exert a modulating effect on the onset age in patients with obsessive-compulsive disorder. Psychoneuroendocrinology. 2017:86:45-52.

18. Cappi C, Diniz JB, Requena GL, Lourenço T, Lisboa BCG, Batistuzzo MC, et al. Epigenetic evidence for involvement of the oxytocin receptor gene in obsessive-compulsive disorder. BMC Neurosci. 2016;17(1):79.

19. Elagoz Yuksel M, Yuceturk B, Karatas OF, Ozen M, Dogangun B. The altered promoter methylation of oxytocin receptor gene in autism. J Neurogenet. 2016;30(3-4):280-4.

20. Altemus M, Jacobson KR, Debellis M, Kling M, Pigott T, Murphy DL, et al. Normal CSF oxytocin and NPY levels in OCD. Biol Psychiatry. 1999;45(7):931-3.

21. Marroni SS, Nakano FN, Gati CD, Oliveira JA, Antunes-Rodriques J, GarciaCairasco N. Neuroanatomical and cellular substrates of hypergrooming induced by microinjection of oxytocin in central nucleus of amygdala, an experimental model of compulsive behavior. Mol Psychiatry. 2007;12(12): 1103-17.

22. Meadows JP, Guzman-Karlsson MC, Phillips S, Holleman C, Posey JL, Day J J et al. DNA methylation regulates neuronal glutamatergic synaptic scaling. Sci Signal. 2015;8(382):ra61-ra.

23. Nelson ED, Kavalali ET, Monteggia LM. Activity-dependent suppression of miniature neurotransmission through the regulation of DNA methylation. Neurosci. 2008;28(2):395-406

24. van den Heuvel OA, Remijnse PL, Mataix-Cols D, Vrenken H, Groenewegen $H J$, Uylings HB, et al. The major symptom dimensions of obsessivecompulsive disorder are mediated by partially distinct neural systems. Brain. 2009;132(Pt 4):853-68.
25. Cadman T, Spain D, Johnston P, Russell A, Mataix-Cols D, Craig M, et al. Obsessive-compulsive disorder in adults with high-functioning autism spectrum disorder: what does self-report with the OCI-R tell us? Autism Res. 2015;8(5):477-85.

26. Ruta L, Mugno D, D’Arrigo VG, Vitiello B, Mazzone L. Obsessive-compulsive traits in children and adolescents with Asperger syndrome. Eur Child Adolesc Psychiatry. 2010;19(1):17.

27. Jacob S, Landeros-Weisenberger A, Leckman JF. Autism spectrum and obsessive-compulsive disorders: OC behaviors, phenotypes and genetics. Autism Res. 2009;2(6):293-311.

28. Lawrence NS, Wooderson S, Mataix-Cols D, David R, Speckens A, Phillips ML. Decision making and set shifting impairments are associated with distinct symptom dimensions in obsessive-compulsive disorder. Neuropsychology. 2006;20(4):409-19.

29. Mataix-Cols D, Rosario-Campos MC, Leckman JF. A multidimensional model of obsessive-compulsive disorder. Am J Psychiatry. 2005;162(2):228-38.

30. Jack A, Connelly JJ, Morris JP. DNA methylation of the oxytocin receptor gene predicts neural response to ambiguous social stimuli. Front Hum Neurosci. 2012;6:280

31. Puglia MH, Connelly JJ, Morris JP. Epigenetic regulation of the oxytocin receptor is associated with neural response during selective social attention. Transl Psychiatry. 2018;8(1):116.

32. Lee KW, Pausova Z. Cigarette smoking and DNA methylation. Front Genet. 2013;4:132.

33. Reinius $L E$, Acevedo N, Joerink M, Pershagen $G$, Dahlén S-E, Greco D, et al. Differential DNA methylation in purified human blood cells: implications for cell lineage and studies on disease susceptibility. PLoS One. 2012;7(7).

34. Abdul QA, Yu BP, Chung HY, Jung HA, Choi JS. Epigenetic modifications of gene expression by lifestyle and environment. Arch Pharm Res. 2017;40(11): 1219-37.

35. First MB, Spitzer R, L GM, Williams JBW. Structured clinical interview for DSM-IV axis I disorders, clinical version (SCID-CV). Washington, D.C. : American Psychiatric Press, Inc.; 1996.

36. Goodman W, Rasmussen S, Price L, Mazure L, Heninger G, Charney D. YaleBrown Obsessive Compulsive Scale (Y-BOCS). Verhaltenstherapie. 1991;1(3): 226-33.

37. Montgomery SA, Asberg M. A new depression scale designed to be sensitive to change. Br J Psychiatry. 1979;134:382-9.

38. Foa EB, Huppert JD, Leiberg S, Langner R, Kichic R, Hajcak G, et al. The Obsessive-Compulsive Inventory: development and validation of a short version. Psychol Assess. 2002;14(4):485.

39. Lim JS, Kim SJ, Jeon WT, Cha KR, Park JH, Kim C-H. Reliability and validity of the Korean version of Obsessive-Compulsive Inventory-Revised in a nonclinical sample. Yonsei Med J. 2008:49(6):909-16.

40. Unternaehrer E, Luers P, Mill J, Dempster E, Meyer AH, Staehli S, et al. Dynamic changes in DNA methylation of stress-associated genes (OXTR, BDNF) after acute psychosocial stress. Transl Psychiatry. 2012;2:e150.

41. Liu W, Fan J, Gan J, Lei H, Niu C, Chan RC, et al. Disassociation of cognitive and affective aspects of theory of mind in obsessive-compulsive disorder. Psychiatry Res. 2017;255:367-72.

42. Misır E, Bora E, Akdede BB. Relationship between social-cognitive and socialperceptual aspects of theory of mind and neurocognitive deficits, insight level and schizotypal traits in obsessive-compulsive disorder. Compr Psychiatry. 2018;83:1-6.

43. Blom G. Statistical estimates and transformed beta-variables. Almqvist \& Wiksell; 1958.

44. Cushing BS, Kramer KM. Mechanisms underlying epigenetic effects of early social experience: the role of neuropeptides and steroids. Neurosci Biobehav Rev. 2005;29(7):1089-105.

45. Dumais KM, Veenema AH. Vasopressin and oxytocin receptor systems in the brain: sex differences and sex-specific regulation of social behavior. Front Neuroendocrinol. 2016;40:1-23.

46. Li K, Nakajima M, Ibañez-Tallon I, Heintz N. A cortical circuit for sexually dimorphic oxytocin-dependent anxiety behaviors. Cell. 2016;167(1):60-72. e11.

\section{Publisher's Note}

Springer Nature remains neutral with regard to jurisdictional claims in published maps and institutional affiliations. 